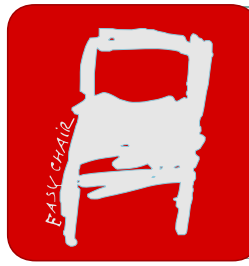

EPiC Series in Health Sciences

Volume 3, 2019, Pages 301-305

CAOS 2019. The 19th Annual Meeting of the International Society for Computer Assisted Orthopaedic Surgery

\title{
Shadow Peak: Accurate Real-time Bone Segmentation for Ultrasound and Developmental Dysplasia of the Hip
}

\author{
Prashant Pandey ${ }^{1}$, Niamul Quader ${ }^{1}$, Kishore Mulpuri ${ }^{2}$, Pierre Guy ${ }^{3}$, Rafeef \\ Garbi $^{1}$, and Antony J. Hodgson ${ }^{4}$ \\ 1 Electrical and Computer Engineering, University of British Columbia Vancouver, BC, Canada \\ prashant@ece.ubc.ca \\ 2 Department of Orthopaedic Surgery, British Columbia Children's Hospital, Vancouver, BC, Canada \\ 3 Department of Orthopaedics, University of British Columbia, Vancouver, BC, Canada \\ 4 Department of Mechanical Engineering, University of British Columbia, Vancouver, BC, Canada
}

\begin{abstract}
Confidence-weighted structured phase symmetry (CSPS) is a state-of-the-art bone segmentation technique for ultrasound (US), which has been recently proposed for automatic diagnosis for developmental dysplasia of the hip (DDH). However, CSPS relies on complex image phase feature analysis which is computationally expensive, and in our preliminary tests we have found it to be sometimes inaccurate. We evaluate a simpler alternative segmentation technique which we previously published, called Shadow Peak (SP), which uses intensity analysis to perform fast and accurate US bone segmentation. On average, SP segmentation ran 15 times faster for 2D US images, when tested on 15 hip images of pediatric patients. Furthermore, SP improves the segmentation F-score to $94 \%$, compared to $72 \%$ when using CSPS segmentation.
\end{abstract}

\section{Introduction}

Ultrasound (US) has been shown to be a reliable, inexpensive and safe imaging modality for orthopaedic applications, with real-time imaging capabilities. In particular, US was used and automatically processed to diagnose developmental dysplasia of the hip (DDH), a pediatric hip disorder, by extracting key 2D and 3D DDH image metrics [7, 3]. US bone segmentation is a key component in this automated diagnosis. Many US bone segmentation techniques exist, including optimized phase symmetry (OPS) and confidence-weighted structured phase symmetry (CSPS) which have been used in DDH image analysis [5, 6]. However, in preliminary experiments we found both OPS and CSPS sometimes inaccurately segmented bone surfaces in US.

We therefore present an alternative bone segmentation technique, Shadow Peak (SP), which uses primarily intensity-based vectorized operations (e.g. scanline integration, array multiplication) to achieve real-time segmentation in US images. Previously, we demonstrated the efficacy of SP segmentation on spatially reconstructed 3D US volumes for computer-assisted orthopaedic surgery applications [4], and here we demonstrate its performance for bone segmentation in 2D in vivo images of pediatric patients. 


\section{Methods}

Shadow Peak (SP) calculates a shadow confidence map based on vectorized normalized scanline integration, which is used to enhance the original US image through element-wise multiplication. Peak detection and connected-component analysis are then used to produce the final bone segmentation. We implemented the method as described in [4], but changed the Gaussian filter's standard deviation from 1 to 4 pixels, given that bone surfaces in DDH images have a larger pixel thickness, compared to our previous study. We implemented CSPS and OPS segmentation using the original authors' respective code, and implemented ray-casting to select the final segmentations as recommended by the authors [7, 2]. We used the same connectedcomponent analysis parameters across all segmentation methods to enable a fair comparison.

We used fifteen US hip volumes, which were made accessible from a previously completed study. The volumes were collected from 11 infant patients at British Columbia Children's Hospital, using a SonixTouch Q+ ultrasound machine (BK Ultrasound, Peabody, MA, USA). No two scans contained the same hip from a patient. The bone in the centre slice from each volume was manually segmented (which we used as the gold standard) by a pediatric orthopaedic surgeon with expertise in infant hip US images. Each US image was resized to an isotropic pixel size of $0.158 \mathrm{~mm}$, represented approximately by $250 \times 250$ pixels. $2 \mathrm{D}$ automatic segmentations using SP, CSPS and OPS were performed on the centre slice of each volume.

To assess the accuracy of the segmentation methods, we measured the recall and precision of each segmentation after 1 or $2 \mathrm{~mm}$ contour dilation, consistent with the method proposed by [1]. We also calculated the F-score for each segmentation, defined as the harmonic mean of the recall and precision. In addition, we measured the root-mean-square (RMS) Euclidean distance error between the automatic and manual segmentations. We computed two Euclidean distances: one going from each point on the automatic segmentation to the nearest manual segmentation point, and the second going from each point on the manual segmentation to the nearest automatic segmentation point. Reporting both distances is more sensitive to discrepancies in the surface extents between automatic and manual segmentations. Lastly, we recorded runtime for each segmentation method.

\section{Results}

Figure 1 shows qualitative results on the DDH images, comparing fair and poor segmentation results for each of the segmentation techniques. In our test, CSPS tended to under-segment the ilium, while OPS sometimes segmented non-bone structures (see Figure 1).

Overall on the $15 \mathrm{DDH}$ images, we found that SP achieved both the highest mean precision and recall: $90.2 \%$ (standard deviation (SD): $11.2 \%$ ) precision and $98.3 \%$ (SD: $5.2 \%$ ) recall, giving an overall F-score of $93.7 \%$ (SD: $7.8 \%$ ). CSPS achieved $85.1 \%$ (SD: $28.3 \%$ ) precision and $66.3 \%$ (SD: $31.7 \%$ ) recall, while OPS achieved $72.9 \%$ (SD: $40.3 \%$ ) precision and $69.7 \%$ (SD: $41.8 \%)$ recall. We tested the significance of these results using a one-tailed Wilcoxon signedrank test and found that Shadow Peak had a mean F-score greater than CSPS $(p<0.01)$ and OPS $(p<0.05)$. The F-scores are plotted in Figure 2. For CSPS segmentation, the mean RMS distance to and from the manual segmentation was $2.06 \mathrm{~mm}$ (SD: $4.24 \mathrm{~mm}$ ) and $2.16 \mathrm{~mm}$ (SD: $4.27 \mathrm{~mm}$ ) respectively. For OPS, the mean RMS distance to and from the manual segmentation was $4.84 \mathrm{~mm}$ (SD: $8.17 \mathrm{~mm}$ ) and $3.94 \mathrm{~mm}$ (SD: $7.46 \mathrm{~mm}$ ). SP achieved the lowest mean RMS distance errors both to and from the manual segmentation: the mean RMS distance from the $\mathrm{SP}$ segmentation to and from the manual segmentation was $1.14 \mathrm{~mm}$ (SD: $0.85 \mathrm{~mm}$ ) and $0.28 \mathrm{~mm}$ (SD: $0.17 \mathrm{~mm}$ ) respectively. 

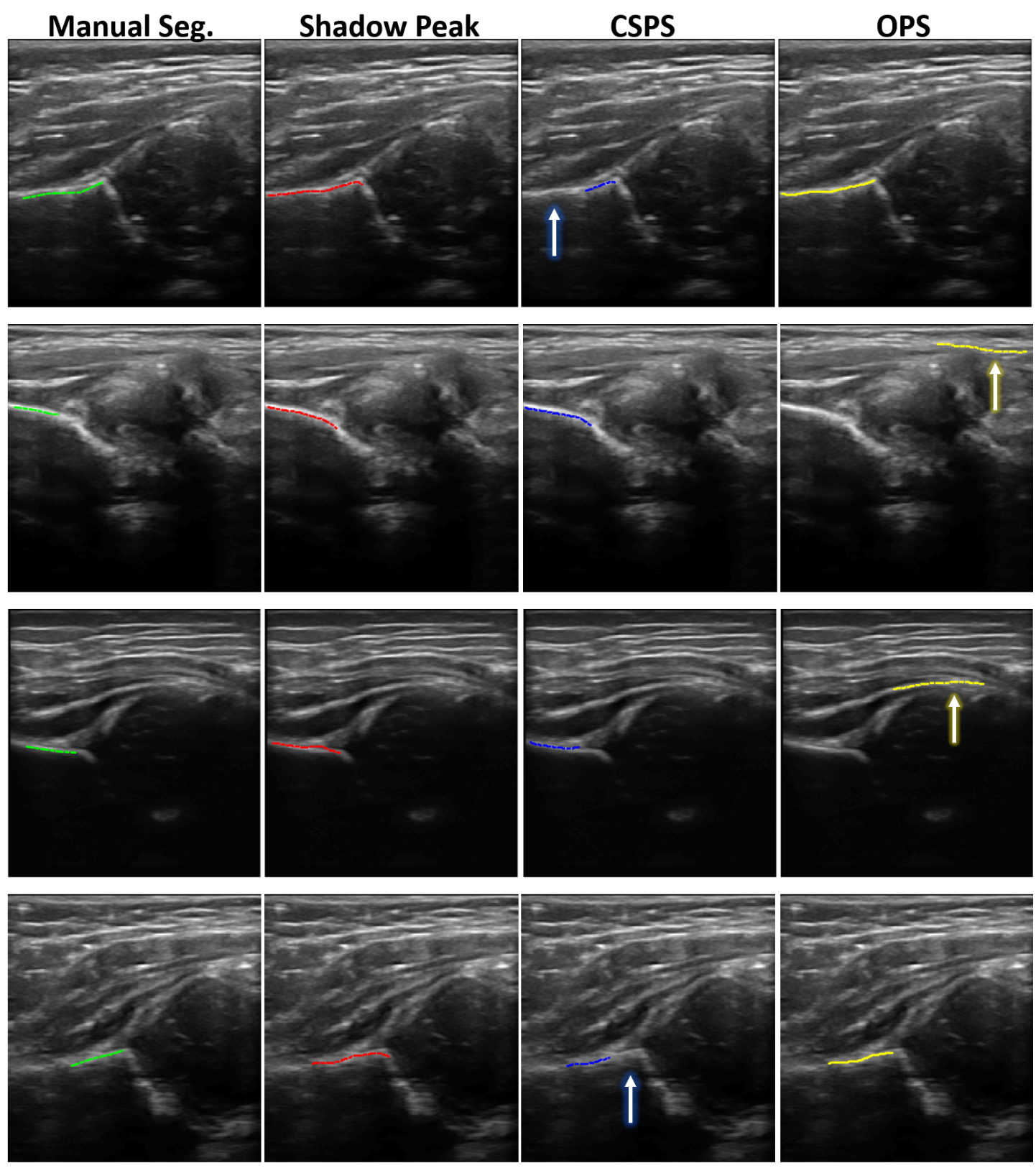

Figure 1: Four example DDH images, with corresponding manual and automatic segmentations. The blue arrows highlight typical bone under-segmentation by CSPS, and the red arrows highlight how incorrect structures are sometimes segmented by OPS. 


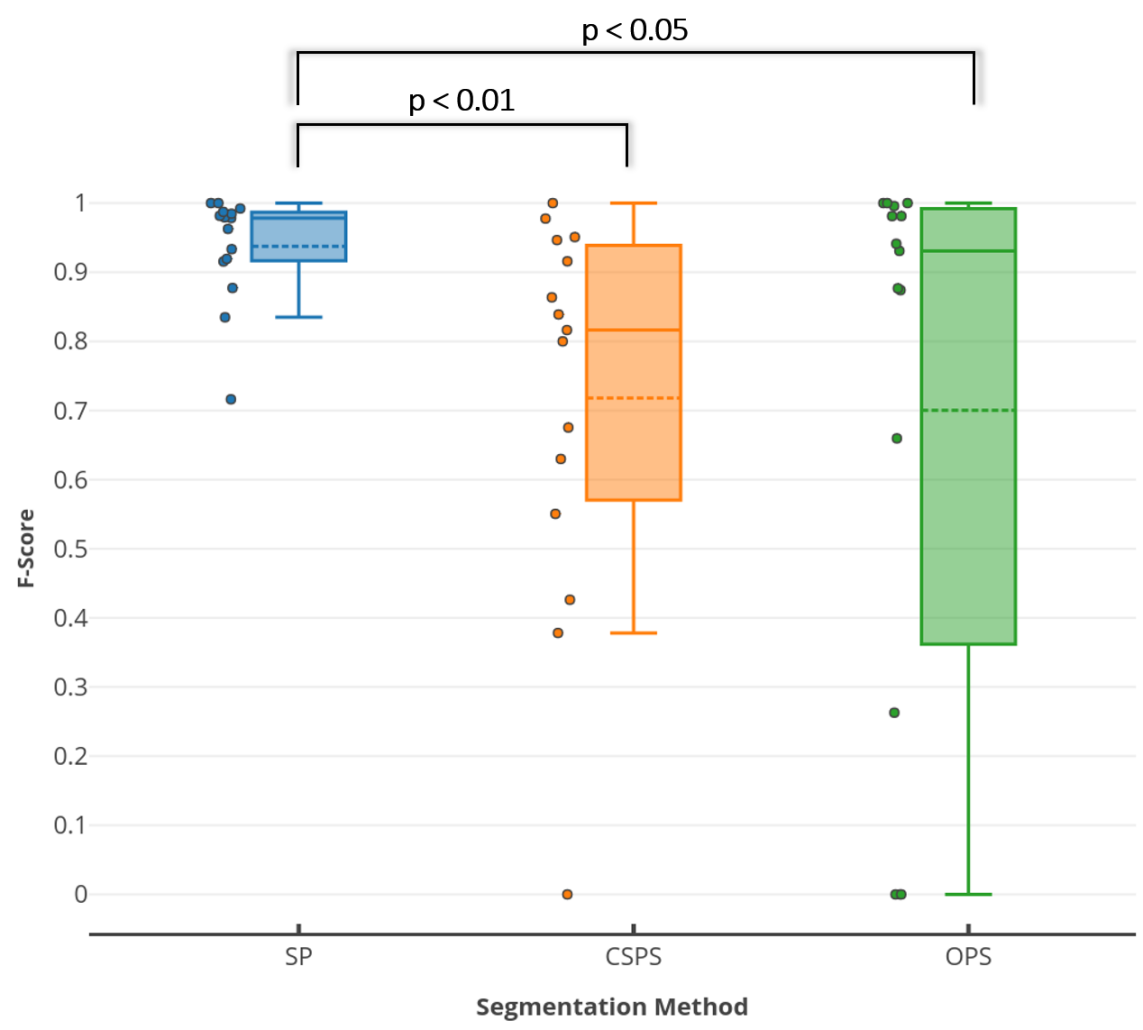

Figure 2: F-scores of each segmentation method evaluated against manual expert segmentation. The dashed line represents the mean and the solid line represents the median in each box plot.

SP took an average of $6 \mathrm{~ms}$ (SD: $2 \mathrm{~ms}$ ) to process a single US image. In contrast, OPS took an average of $450 \mathrm{~ms}$ (SD: $22 \mathrm{~ms}$ ) and CSPS segmentation took an average of $94 \mathrm{~ms}$ (SD: $7 \mathrm{~ms}$ ) to compute. The difference in mean runtimes across the three methods are statistically significant using a student's t-test $(p<0.0001)$. All segmentations were performed in MATLAB on an Intel i7-6700HQ CPU with 32GB RAM.

\section{Discussion and Conclusion}

We found that SP segmentation was both more accurate and faster than CSPS and OPS for bone in $2 \mathrm{D}$ in vivo images of the pediatric hip. We found comparable performance in terms of the absolute F-score when compared to other state-of-the-art methods - in particular the deep learning based methods presented in [1] and [8] achieved mean F-scores of 0.90 and 0.64 respectively, whereas SP achieved 0.94. However, it is challenging to compare results directly given the different anatomies imaged in each study. One limitation of our study is that bone segmentation accuracy was assessed on a small set of patient images, and a more representative assessment could be made on images collected from more patients and using different US imaging systems.

Given the higher F-score and lower RMS errors, we believe that SP segmentation has the 
potential to make automated DDH diagnoses more efficient and accurate. Furthermore, we plan to study the effect of SP segmentation on the reliability of automated DDH diagnoses, when used directly on 3D US volumes.

\section{References}

[1] Nora Baka, Sieger Leenstra, and Theo van Walsum. Ultrasound aided vertebral level localization for lumbar surgery. IEEE Trans. Med. Imaging, 36(10):1-1, 2017.

[2] Ilker Hacihaliloglu, Rafeef Abugharbieh, Antony J. Hodgson, and Robert N. Rohling. Automatic Adaptive Parameterization in Local Phase Feature-Based Bone Segmentation in Ultrasound. Ultrasound Med. Biol., 37(10):1689-1703, 2011.

[3] Abhilash R. Hareendranathan, Dornoosh Zonoobi, Myles Mabee, Dana Cobzas, Kumaradevan Punithakumar, Michelle Noga, and Jacob L. Jaremko. Toward automatic diagnosis of hip dysplasia from 2D ultrasound. In 2017 IEEE 14th Int. Symp. Biomed. Imaging (ISBI 2017), pages 982-985. IEEE, apr 2017.

[4] Prashant Pandey, Pierre Guy, Antony J Hodgson, and Rafeef Abugharbieh. Fast and automatic bone segmentation and registration of 3D ultrasound to CT for the full pelvic anatomy: a comparative study. Int. J. Comput. Assist. Radiol. Surg., may 2018.

[5] Niamul Quader, Antony Hodgson, and Rafeef Abugharbieh. Assessing the Feasibility of Downsampling and Wavelet Resizing for Real-Time Extraction of Bone Surfaces From 3D Ultrasound. Comput. Assist. Orthop. Surg., 2015.

[6] Niamul Quader, Antony J. Hodgson, Kishore Mulpuri, and Rafeef Abugharbieh. Improving Diagnostic Accuracy of Hip Dysplasia Measures in 2D Ultrasound Scans of Infants to Guide Decisions Regarding Need For Surgery. Comput. Assist. Orthop. Surg., 2015.

[7] Niamul Quader, Antony J Hodgson, Kishore Mulpuri, Anthony Cooper, and Rafeef Abugharbieh. A 3D Femoral Head Coverage Metric for Enhanced Reliability in Diagnosing Hip Dysplasia. MICCAI 2017, 10433:100-107, 2017.

[8] M. Villa, G. Dardenne, M. Nasan, H. Letissier, C. Hamitouche, and E. Stindel. FCN-based approach for the automatic segmentation of bone surfaces in ultrasound images. Int. J. Comput. Assist. Radiol. Surg., 13(11):1707-1716, nov 2018. 